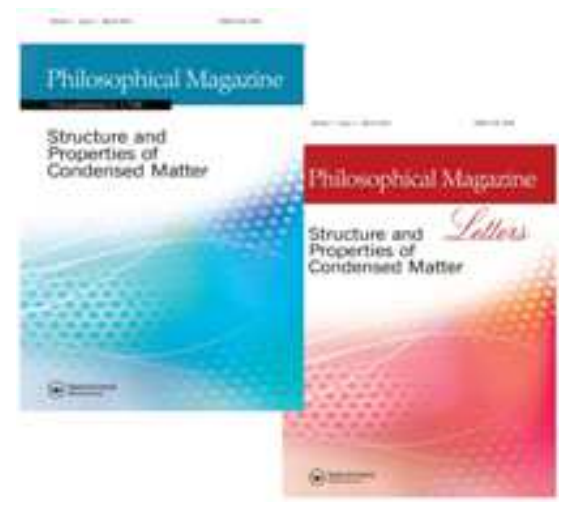

\title{
Nucleation pathway in coherent precipitation
}

\begin{tabular}{|r|l|}
\hline Journal: & Philosophical Magazine \& Philosophical Magazine Letters \\
\hline Manuscript ID: & TPHM-11-May-0173.R2 \\
\hline Journal Selection: & Philosophical Magazine \\
\hline Date Submitted by the \\
Author: & $17-$ Aug-2011 \\
\hline Complete List of Authors: & $\begin{array}{l}\text { Philippe, Thomas; GPM } \\
\text { Blavette, Didier; GPM }\end{array}$ \\
\hline Keywords: & solid-state transformation, precipitation, nucleation \\
\hline Keywords (user supplied): & minimum energy pathway \\
\hline &
\end{tabular}

\section{SCHOLARONE}

Manuscripts 


\section{Nucleation pathway in coherent precipitation}

T. Philippe ${ }^{1} \dagger$ and D. Blavette ${ }^{1 *}$

${ }^{1}$ Université de Rouen, GPM, UMR CNRS 6634 BP 12, Avenue de l'Université 76801

Saint Etienne de Rouvray, France

* Institut Universitaire de France, France

$†$ Contact author: thomas.philippe@etu.univ-rouen.fr 


\section{Nucleation pathway in coherent precipitation}

The non-classical nucleation pathway of coherent precipitates has been computed through the minimisation of the nucleation barrier in the composition (c) - size (R) space so that to predict the evolution of the nucleus composition. The generalized Gibbs model developed by Schmelzer et al. has been extended so that to include the misfit elastic energy. The composition of critical embryos $c^{*}$ has been found independent on the interfacial constant. The composition of critical nuclei $\left(\mathrm{c}^{*}\right)$ was found to decrease with supersaturation. The elastic energy increases both $\mathrm{c}^{*}$ and the nucleation barrier as well as $\mathrm{R}^{*}$. The evolution of the nucleus composition (c) as a function of its size (R) along the minimum energy pathway was computed. Nucleation only starts when exceeding a size threshold. Then, a rapid enrichment up to the expected composition $\left(\mathrm{c}_{\beta}\right)$ precedes a constant composition regime. However, for small supersaturation, the change in cluster composition can occur sharply for very small radius and then the composition slowly increases with a significant change in size. Coherency misfit energy was found to slow down the evolution of the nuclei composition with R. Model was confronted to experiments.

Keywords: solid state phase transformations, precipitation, non-classical nucleation, minimum energy pathway

\section{Introduction}

Numerous solid state transformations among them precipitation proceed via first-order phase transitions. According to the supersaturation, the early stages of precipitation in alloys can be classically accounted for either by nucleation and growth of embryo or spinodal decomposition for which no barrier is involved. The early developments of the thermodynamics of first-order phase transitions were performed by Gibbs [1] and van der Waals [2,3]. The first formulation of the nucleation-growth model was done by Gibbs [4-9]. Cahn and Hilliard re-expressed the van der Waals method to develop a continuum theory of nucleation $[10,11]$. Many developments and refinements were brought about to the early theories of pioneers like Becker [12]. Let us mention the models of Zeldovich and Turnbull which provide us with a latent nucleation time $[13,14]$. 
When the equilibrium phase has a different structure as the parent solid solution, the system often prefers to form a transient coherent phase that has the same structure as the initial solid solution (coherent nucleation). This is well known in Al base alloys, notably in $\mathrm{AlCu}$ where coherent GP zones nucleate prior the precipitation of the equilibrium phase $\mathrm{Al}_{2} \mathrm{Cu}$ [15]. It is well accepted that these GP zones are almost pure in copper. However, in other systems, and outside the spinodal region, dilute and diffuse clusters are observed to form before the equilibrium phase precipitates. Such nonclassical nucleation processes were observed both in metals (Fe-Cu, Fe-Cr) and in semiconductors (Si-B, Si-As) using atom probe tomography (APT) [16,17]. Because of its ultimate spatial resolution, APT has played a major role in the investigation of the early stages of decomposition [18-31]. Although the system is in a metastable state (e.g. outside the spinodal regime of decomposition where the system is in an unstable state), nuclei were observed with a lower solute content than the equilibrium phase and found to enrich with time before reaching the expected composition. Interfaces sometimes appear diffuse. Diffusion as well as thermodynamics control kinetics pathway during precipitation [32-34]. In this paper, diffusion effects have been ignored. Only arguments based on thermodynamics are taken into account.

The nucleation of embryos that have a low solute content may appear surprising when considering the only contribution of the driving force for nucleation $\Delta \mathrm{f}$. This force is clearly shown in figure 1 to decrease when the solute content in the nucleus is smaller. However, this can be counterbalanced by the lower interfacial energy $(\sigma)$ that occur between diffuse nuclei and the parent phase. $\sigma$ is indeed proportional to the concentration gradient $(\nabla \mathrm{c})^{2}$ at the interface. The nucleation barrier, proportional to $\sigma^{3}$, 
is consequently much lower for dilute and diffuse nuclei. Dilute nuclei also leads to a smaller mismatch between phases that reduces the misfit elastic energy that also acts against nucleation. In other terms, in spite of the lower driving force, the barrier of nucleation of such dilute and diffuse solute-enriched zones can be lower than that of the expected coherent phase. This article aims at predicting the optimum composition of nuclei that minimises the nucleation barrier. Kinetics arguments related to diffusion were not considered in this approach. Another question to which this article wishes to answer is also how the composition of nuclei evolves towards equilibrium with increasing size.

The diffuse interface theory of non-classical nucleation developed by Cahn and Hilliard has been the first to deal with this issue $[10,11]$. The theory is based on the determination of the radial concentration profile of the critical nucleus minimizing the nucleation barrier. They found that the classical nucleation theory was only operative for small supersaturation. Composition profile of a critical nucleus as a function of the nominal composition $\left(\mathrm{c}_{0}\right)$ from the equilibrium composition $\left(\mathrm{c}_{\alpha}\right)$ to the spinodal one $\left(\mathrm{c}_{\mathrm{s}}\right)$ was investigated in regular solutions $(\mathrm{T} / \mathrm{Tc}=0.8656)$. They found that the nucleus composition (at the core of the nucleus) approaches the matrix composition near the spinodal line. The critical radius of nucleation was found to diverge to infinity not only near $\mathrm{c}_{\alpha}$ but also close to the spinodal line $\mathrm{c}_{\mathrm{s}}$. The strong point of this diffuse interface theory is that no hypothesis about the chemical homogeneity of nuclei is required. More recently, Schmelzer et al. developed a simpler model (the generalized Gibbs theory) based on the same ideas as Cahn and Hilliard, i.e the minimization of the nucleation barrier [35]. In contrast to Cahn, the authors considered nuclei with an homogeneous 
composition. However, the elastic energy related to misfit stress between phases was not considered despite of its crucial importance for precipitation in solids.

In this work, we have focused on the nucleation of precipitates in solids. We have therefore extended the generalized Gibbs model developed by Schmelzer et al. $[35,36]$ so that to include the contribution of the misfit elastic energy [37]. The latters have studied the influence of elastic stress on crystallisation in glasses [38]. Here, we have focused on the influence of misfit on solid state precipitation. We have confronted predictions to experiment results provided by atom-probe tomography. The latter is one of the very rare instruments able to measure the composition of small embryo $(1 \mathrm{~nm})$ in the early stages of nucleation. The model is only based on thermodynamics arguments. The influence of diffusion and coupling effects of diffusion fluxes on the kinetics pathway was not considered. Nucleation was modelled as a function of the composition and radius of nucleus. The composition of embryo was assumed to be uniform and was not constrained to be that of the equilibrium phase contrary to the classical nucleation theory. The chemical composition $\left(\mathrm{c}^{*}\right)$ of the critical nucleus as a function of the nominal composition was computed through the determination of the saddle point of the nucleation barrier $\mathrm{W}(\mathrm{R}, \mathrm{c})$ in the $\mathrm{R}, \mathrm{c}$ space. A regular solution was considered in order to compute the driving force for nucleation. Isotropic elastic energy was considered (Eshelby theory of inclusions [37]). The most probable trajectory, the minimum energy pathway, along the free energy surface $\mathrm{W}(\mathrm{R}, \mathrm{c})$ was computed numerically. This made it possible to predict how the composition evolves as a function of the embryo size in the early stages of nucleation. Confrontation of predictions to experiments dealing with the early stages of precipitation in $\mathrm{Si}-\mathrm{B}, \mathrm{Fe}-\mathrm{Cr}$ and $\mathrm{Fe}-\mathrm{Cu}$ systems has been carried out. 


\section{Determination of saddle point}

\section{a. Model}

Let us consider the nucleation of B-enriched precipitates in A-B binary solid solution $(\alpha)$. The contribution of the misfit elastic energy has been considered. Interfacial and elastic energies have been assumed to be isotropic. The nucleus composition has been assumed to be uniform. The nucleation of the new phase called hereafter $\beta$ has been investigated as a function of the size (spherical nucleus of radius $\mathrm{R}$ ) and composition (nucleus composition c). As for classical nucleation theory, the composition of the parent solid solution was assumed to be nearly constant during nucleation (negligible volume fraction of $\beta$ phase). The free energy change due to the formation of a nucleus can be classically expressed as:

$$
\Delta F=-(\Delta f-\gamma) V+\sigma S
$$

$\Delta f$ (in $\mathrm{J} / \mathrm{m}^{3}$ ) is the driving force for nucleation (Fig.1) is defined as following:

$$
\Delta f=f\left(c_{0}\right)+\left.\left(c-c_{0}\right) \frac{\partial f}{\partial c}\right|_{c=c_{0}}-f(c)
$$

where $\mathrm{c}$ is the nucleus composition (i.e. atomic fraction of $\mathrm{B}$ ), $\mathrm{c}_{0}$ the nominal one and the function $\mathrm{f}$ is the free energy (fig.1). For the sake of simplicity, an isotropic inclusion has been considered. The elastic energy $\gamma$, as given by Eshelby [37] can be written as proportional to the square function of the lattice misfit between the nucleus $\beta$ and the parent phase $\alpha$. The misfit being proportional to the composition difference between both phases (Vegard law), one can simply express $\gamma$ as:

$$
\gamma=\tilde{\gamma}\left(c-c_{0}\right)^{2}
$$


with $\tilde{\gamma}=\mathrm{E} . \delta^{2} /(1-v)$, with $\mathrm{E}$ the Young modulus, $v$ the Poisson coefficient and $\delta$ the misfit between pure phases $\left(\delta=\delta a / a\right.$, with a the average lattice parameter and $\delta a=a_{B}-$ $\left.a_{A}\right)$.

The interfacial energy was written as proportional to the concentration gradient squared:

$$
\sigma=\tilde{\sigma}\left(c-c_{0}\right)^{2}
$$

with $\tilde{\sigma}=\varepsilon / \mathrm{d}^{2}, \varepsilon$ the pair interaction energy and $\mathrm{d}$ the interface thickness. This latter parameter (d) may account for the diffuseness of interfaces.

Nuclei were assumed to be spherical (radius R) so that the change of free energy reads:

$$
\Delta F=-\frac{4 \pi}{3} R^{3}\left(\Delta f-\tilde{\gamma}\left(c-c_{0}\right)^{2}\right)+4 \pi R^{2} \tilde{\sigma}\left(c-c_{0}\right)^{2}
$$

The free energy surface, $\Delta \mathrm{F}(\mathrm{R}, \mathrm{c})$, for a given nominal composition $\left(\mathrm{c}_{0}\right)$ acts as a thermodynamic barrier over which the growing nucleus must pass through before growth. The major nucleation flux generally passes through the saddle point [12]. The location of this saddle point is defined by the two following equations:

$$
\begin{gathered}
\frac{\partial \Delta F}{\partial R}=0 \Leftrightarrow R=\frac{2 \tilde{\sigma}\left(c-c_{0}\right)^{2}}{\Delta f-\tilde{\gamma}\left(c-c_{0}\right)^{2}} \\
\frac{\partial \Delta F}{\partial c}=0 \Leftrightarrow-\frac{R}{3}\left(\frac{\partial \Delta f}{\partial c}-2 \tilde{\gamma}\left(c-c_{0}\right)\right)+2 \tilde{\sigma}\left(c-c_{0}\right)=0
\end{gathered}
$$

The composition of the critical nucleus $\left(\mathrm{c}^{*}\right)$ can be derived by combining equations (6) and (7): 


$$
\left.\frac{\partial \Delta f}{\partial c}\right|_{c=c^{*}}+\tilde{\gamma}\left(c^{*}-c_{0}\right)=\frac{3 \Delta f}{c^{*}-c_{0}}
$$

The expression of $\Delta \mathrm{f}$ (section $\mathrm{b}$ ) makes it possible to find $\mathrm{c}^{*}$. Once the critical composition $\left(\mathrm{c}^{*}\right)$ is determined, the critical radius $\left(\mathrm{R}^{*}\right)$ can be derived from equation (6). It is worth mentioning that equation (8) does not depend on the interfacial energy factor $\tilde{\sigma}$. As a result, surprisingly, $\mathrm{c}^{*}$ is independent of $\tilde{\sigma}$ (eq.8). The work required to form a nucleus (nucleation barrier W) is defined as the free energy change associated with the formation of a critical nucleus $\left(\mathrm{R}^{*}, \mathrm{c}^{*}\right)$ :

$$
W=\Delta F\left(c^{*}, c_{0}\right)=\frac{16 \pi}{3} \frac{\tilde{\sigma}^{3}\left(c^{*}-c_{0}\right)^{6}}{\left(\Delta f-\tilde{\gamma}\left(c^{*}-c_{0}\right)^{2}\right)^{2}}
$$

As the interfacial term of $\mathrm{W}$ grows as the $6^{\text {th }}$ power of the nucleus composition, it may be anticipated that the nucleation of nuclei of low solute content (smaller W) may be favoured although the driving force is smaller for such dilute nuclei. The model will be applied in the next section to a regular solution for the sake of simplicity. More realistic functional can however be used.

\section{b. Non-classical nucleation in a regular solution}

Let us consider a regular solution subject to unmixing. The free energy (f) can be expressed as:

$$
\frac{f}{k_{B} T}=\frac{2}{\eta} c(1-c)+c \ln c+(1-c) \ln (1-c)
$$

where $\mathrm{c}$ is the atomic fraction of solute atoms and $\eta$ the reduced temperature defined as $T / T_{c}$ with $T$ the temperature and $T_{c}$ the critical temperature. The critical temperature $T_{c}$ is related to the interaction parameter $\varepsilon$ by 


$$
T_{c}=\frac{Z \varepsilon}{2 k_{B}}
$$

with $\mathrm{Z}$ the coordinance and $\varepsilon$ the energy of ordering classically expressed as a function of effective pair interaction energies between first nearest neighbour atoms (AA, BB, $\mathrm{AB})$.

The binodal line $\left(\mathrm{df} / \mathrm{dc}=0-\right.$ solubility limits $\left.\mathrm{c}_{\alpha}\right)$ and the spinodal line $\left(\mathrm{c}_{\mathrm{s}}\right.$, $\left.d^{2} f / d c^{2}=0\right)$ in the $(\eta, c)$ phase diagram are given respectively by:

$$
\begin{gathered}
\ln \left(\frac{1-c_{\alpha}}{c_{\alpha}}\right)=\frac{2}{\eta}\left(1-2 c_{\alpha}\right) \\
c_{s}\left(1-c_{s}\right)=\frac{\eta}{4}
\end{gathered}
$$

Both curves classically coincide at the critical point $(\eta=1, c=0.5)$. As an illustration, let us choose a system for a reduced temperature $\eta=0.3$ (solubility limit $\left.\mathrm{c}_{\alpha} \sim 0.0013\right)$. The spinodal curve gives $\mathrm{c}_{\mathrm{s}} \sim 0.0816$. We have focused on the range $\mathrm{c}_{\alpha}<\mathrm{c}_{0}<\mathrm{c}_{\mathrm{s}}$ where precipitation proceeds via nucleation (spinodal decomposition has not been considered). The driving force for the formation of a nucleus with a given composition $\mathrm{c}$ $\left(\Delta \mathrm{f}(\mathrm{c})\right.$, Fig. 1) can be computed as a function of the nominal composition $\left(\mathrm{c}_{0}\right)$.

Combining equations (2) and (10) leads to:

$$
\begin{gathered}
\Delta f=\rho k_{B} T \cdot \Gamma\left(c_{0}, c\right) \\
\text { with } \Gamma\left(c, c_{0}\right)=\frac{2}{\eta}\left(c-c_{0}\right)^{2}-\left(c \ln \frac{c}{c_{0}}+(1-c) \ln \frac{1-c}{1-c_{0}}\right)
\end{gathered}
$$

where $\rho$ is the atomic density $\left(\mathrm{at} / \mathrm{m}^{3}\right)$. Note that the driving force does not depend on the free energy of pure $\mathrm{A}, \mathrm{B}$ components $\left(\mathrm{F}_{\mathrm{A}}\right.$ and $\left.\mathrm{F}_{\mathrm{B}}\right)$. In the classical nucleation theory, 
$\mathrm{c}^{*}=\mathrm{c}_{\beta}$ (right part of the binodal curve). Here, the composition of the nucleus is not constrained to be that of the equilibrium coherent phase $\left(\mathrm{c}_{\beta}\right)$. The consequence is that we do not consider any local equilibrium at the $\alpha / \beta$ interface at stated in classical growth theories. We consider that the nucleus composition is the one that minimises the nucleation barrier. However, all values of $\mathrm{c}^{*}$ are not valid. Indeed, a phase separation may only occur if the thermodynamic driving force of the transformation $\Delta \mathrm{f}$ is strickly positive (fig. 1). The minimum value ( $\mathrm{c}_{\mathrm{m}}$, fig. 1$)$ of $\mathrm{c}^{*}$ is given by $\Delta \mathrm{f}=0$, i.e. $\Gamma\left(\mathrm{c}, \mathrm{c}_{0}\right)=0$ (eq.14 and 15). The latter equation has no analytical solution. For a given initial composition, $\mathrm{c}_{\mathrm{m}}$ increases when $\mathrm{T} / \mathrm{Tc}$ increases. Once the composition of the critical nucleus $\mathrm{c}^{*}$ is determined (equation (8)), the expressions of $\mathrm{W}$ and $\mathrm{R}^{*}$ can be derived for $\mathrm{c}=\mathrm{c} *$ from equation (6) and (9).

Properties of the critical clusters as predicted by this model are shown and compared to predictions of the classical nucleation theory in Fig.2. We will first examine the situation where the elastic contribution is null $(\gamma=0-$ null misfit $)$. The composition of the critical cluster is observed to drastically decrease with the initial solute concentration $\mathrm{c}_{0}$ (Fig.2a). For small supersaturations $\left(\mathrm{c}_{0}\right.$ close to $\mathrm{c}_{\alpha}$ ), $\mathrm{c}^{*}$ is close to equilibrium $\left(\mathrm{c}_{\beta} \sim 1\right)$ as stated in the classical theory. It decreases with increasing values of $\mathrm{c}_{0}$ and tends towards $\mathrm{c}_{0}$ when approaching the spinodal line $\left(\mathrm{c}_{0} \sim \mathrm{c}_{\mathrm{s}}\right)$. The driving force for nucleation (equation (14)) tends to zero when the spinodal curve is approached (fig. 2.b) whereas it reaches a maximum in the classical case. This result $(\Gamma=0)$ was expected as $\mathrm{c}^{*}$ tends toward $\mathrm{c}_{0}$ when approaching the spinodal line. The work, $\mathrm{W}$, required to form a critical nucleus (Fig. $2 \mathrm{c}$, with $16 \pi \sigma^{3} / 3\left(\rho \mathrm{k}_{\mathrm{B}} \mathrm{T}\right)^{2}=2.33$ and $2 \sigma / \rho \mathrm{k}_{\mathrm{B}} \mathrm{T}=12.7$ ) is obviously lower than the classical nucleation barrier. $\mathrm{W} \rightarrow 0$ when the initial solute concentration approaches the spinodal line. As a result, the critical radius tends to 
It should be kept in mind that nucleation in solids may only occur if the driving force is larger than the elastic energy so that to ensure that the critical radius remains positive. In the classical case, this condition leads to an additional constraint on the nominal composition $\mathrm{c}_{0}$ that should be larger than a minimum value given by:

$$
\rho k_{B} T \Gamma\left(c_{\beta}, c_{0}\right)-\tilde{\gamma}\left(c_{\beta}-c_{0}\right)^{2}>0
$$

The properties of critical clusters are represented as a function of the initial solute concentration in Fig.2 for different value of the elastic constant k $\left(k=\tilde{\gamma} / \rho k_{B} T\right)$. $\underline{\mathrm{k}=1,2 \text { have been used to study the influence of relatively high misfit on the properties of }}$ critical clusters. Typically, $\mathrm{k}=1$ corresponds to a misfit of $\sim 5.6 \%$ and $\mathrm{k}=2$ to a misfit of $\sim 7.9 \%\left(\mathrm{E}=130 \mathrm{GPa}, \rho=5 * 10^{22} \mathrm{at} / \mathrm{cm}^{3}\right.$ and $\left.\mathrm{T}=873 \mathrm{~K}\right)$. Again, for small supersaturation ( $\mathrm{c}_{0}$ small), $\mathrm{c}^{*}$ tends to equilibrium $\left(\mathrm{c}_{\beta}\right)$. The minimum composition $\mathrm{c}_{0}$ required for nucleation to start (positive effective driving force, equation (16)) is thus equivalent to the classical one. This composition corresponds to the solubility limit as given by the coherent bimodal composition (close to 0.01 for $\mathrm{k}=2$, i.e., the coherent $\mathrm{T} / \mathrm{T}_{\mathrm{c}}$ is equal to 0.43). As shown in Fig.2a, the composition of the critical cluster increases drastically when the elastic energy parameter $\mathrm{k}$ increases. In other terms, the minimization of the 
nucleation barrier occurs for greater $\mathrm{c}^{*}$ when increasing the coherency misfit. This could appear as paradoxical as larger values of $\mathrm{c}^{*}$ increase the elastic energy and as a result the nucleation barrier (equation (9)). However, in the present non-classical theory, the effective driving force also depends on $\mathrm{c}^{*}$ and may win in the competition. Moreover, as the addition of the elastic energy corresponds to an increasing of $\mathrm{T} / \mathrm{T}_{\mathrm{c}}$, the minimal composition of the critical cluster, $\mathrm{c}_{\mathrm{m}}$, increases. In contrast to the classical theory, the elastic energy influences the composition of embryo that in turn has a direct influence on the driving force. The chemical driving force $\Gamma\left(\mathrm{c}^{*}\right)$ increases with the elastic energy because $\mathrm{c} *$ increases (Fig.2b). The work required to form a critical nucleus (W) also increases with the elastic energy. It was also find that W vanishes and $\mathrm{R}^{*}$ diverges when the initial solute concentration approaches the coherent spinodal line (Fig.2c and 2d). The coherent spinodal defined as $\mathrm{d}^{2} \mathrm{f} / \mathrm{dc}^{2}=-2 \tilde{\gamma}$ is located inside the incoherent spinodal line $\left(d^{2} \mathrm{f} / \mathrm{dc}^{2}=0\right)$, i.e. the spinodal limit is found for higher concentrations (minimum of W in Fig.2c). As already demonstrated by Cahn and Hilliard, the classical nucleation theory tends to overestimate the work of formation of the critical nucleus. This difference could have a great influence on the calculation of the number density of nuclei $\left(/ \mathrm{m}^{3}\right)$ as it depends on the exponential function of W.

\section{Minimum energy pathway of nucleation}

We propose here to determine the properties of the sub- and supercritical clusters. For that purpose, we consider that the evolution of the cluster properties $(\mathrm{R}, \mathrm{c})$ proceed along a valley of the thermodynamic potential (Fig.3, i.e the 2D nucleation barrier). At the saddle point of the free energy surface $W(R, c)$, the direction of negative curvature is related to the unstable variable (here $\mathrm{R}$ ), while the direction of positive curvature is associated to the stable one (c). Whereas for many problems of physics the 
Let us now consider the dimensionless reduced energy landscape $\Phi(\mathrm{r}, \mathrm{c})$ defined as :

$$
\Phi(r, c)=\frac{\Delta F(r, c)}{W}
$$

where $\mathrm{W}$ is the height of the nucleation barrier at the saddle point. Let us also introduce the reduced radius $r=R / R^{*}$. For a given composition of the system $\left(c_{0}\right)$, $\Phi$ defines the landscape of the energy barrier (fig. 3). Using equations (1) (5), (6), (9) and (17), the function $\Phi$ can finally be written as:

$$
\Phi(r, c)=-2 r^{3} \frac{\Delta f\left(c, c_{0}\right)-\tilde{\gamma}\left(c-c_{0}\right)^{2}}{\Delta f\left(c^{*}, c_{0}\right)-\tilde{\gamma}\left(c^{*}-c_{0}\right)^{2}}+3 r^{2} \frac{\left(c-c_{0}\right)^{2}}{\left(c^{*}-c_{0}\right)^{2}}
$$

Surprisingly, $\Phi(\mathrm{r}, \mathrm{c})$ does not depend explicitly on the interfacial constant. There is however an implicit dependence of $\Phi$ as a function of $\mathrm{R}$ as $\mathrm{r}=\mathrm{R} / \mathrm{R}^{*}$ and $\mathrm{R}^{*}$ is controlled by $\sigma$. It is worth mentioning that there is no analytical expression of the minimum energy pathway (MEP) through such a barrier $\Phi(r, c)$. The MEP was therefore computed numerically from a steepest descent method. 
The function $\Phi$ for a regular solution $\left(\eta=0.3\right.$ and $\left.c_{0}=0.04\right)$ has been plotted in Fig.3 (no elastic energy). The kinetic pathway has to pass through the saddle point ( $\mathrm{r}=1$ and e.g. $\mathrm{c}=\mathrm{c}^{*}=0.456$ for $\left.\mathrm{c}_{0}=0.04\right)$. The nucleation pathway in $(\mathrm{R}, \mathrm{c})$ space for $\eta=0.3$, as a function of supersaturation, was first investigated for $\mathrm{k}=0$ (no misfit between phases). For $\mathrm{c}_{0}=0.04$, a process in three steps is exhibited (fig. 4). The cluster composition stays equal to the initial solute concentration up to $R_{s}=6$. This means that nucleation only starts at $\mathrm{R}_{\mathrm{S}}=6$. For $6<\mathrm{R}<8$, the cluster composition sharply increases up to the expected value $(\mathrm{c}=1)$. The change in size $(\mathrm{R})$ related to this sharp enrichment of embryos is rather small. For $\mathrm{R}>8$, the cluster grows without change of composition. The trajectory for $\mathrm{c}_{0}=0.07, \mathrm{c}_{0}=0.03$ and $\mathrm{c}_{0}=0.02$ are similar (Fig.4). This non-classical model of nucleation gives rise to unanticipated effects. Surprisingly, nucleation is indeed observed to start for smaller radius $\mathrm{R}$, when the supersaturation decreases (i.e. $\mathrm{c}_{0}$ decreases). This is the consequence of the modification of the topology of the barrier $\Phi(r, c)$ (fig. 3) when changing the nominal composition. This modification, that is the result of computation, is complex and is almost impossible to foresee. This three-step nucleation process has already been found by Schmelzer et al. for $\eta=0.7$ [36]. However our results show that this three-step process is not universal. Indeed, for smaller supersaturation $\left(c_{0}=0.01\right.$ and $\eta=0.3$ in Fig. 4 ), there is no more plateau before nucleation starts. The cluster composition increases sharply for $\mathrm{c} \sim 0.46$ and for $\mathrm{R}$ close to zero. After this step, the composition slowly increases towards the equilibrium value.

We shall now examine the influence of the misfit on the minimum energy pathway for various values of the elastic energy parameter $(\mathrm{k}=0,1,2)$. For the same temperature, $\eta=0.3$ and nominal composition $\left(c_{0}=0.04\right.$, Fig. 5$)$ the lattice misfit is shown 
to slow down the evolution of the nuclei composition with $\mathrm{R}$. Increasing $\mathrm{k}$ increases the time required to reach the expected composition $\mathrm{c}=1(\mathrm{k}=0,1)$. However increasing further $\mathrm{k}\left(\mathrm{k}=2\right.$, Fig.5) reduces the onset size $\left(\mathrm{R}_{\mathrm{s}}\right)$ for nucleation. A similar behaviour was found for $\mathrm{c}_{0}=0.02$ (Fig.6). For increasing misfit, the size required for the embryo to reach its asymptotic composition increases.

\section{Model versus experiments}

There are several systems where experiments show that during the early stages of precipitation, nuclei do not have the expected composition. This is the case of $\mathrm{Fe}-\mathrm{Cu}$ [47-51] and $\mathrm{Fe}-\mathrm{Cr}$ [52] where temporal evolution of the nuclei composition has been observed. In boron implanted silicon where the expected precipitated phase has a different structure compared to the diamond lattice of silicon, very dilute boronenriched clusters (7-20 at.\% of boron - 1-5 $\mathrm{nm}$ in size), thought to be coherent with silicon, were evidenced [16,53-55]. This suggests that the problem is general and not simply limited to the early stage of precipitation in metallic alloys. We shall apply the present model to theses systems in order to highlight whether thermodynamic considerations (i.e. minimization of the nucleation barrier) can explain the nucleation of non equilibrium transient coherent clusters during the early stages of precipitation.

Dilute boron-enriched clusters were observed in implanted silicon. Due to the quite different structure of the $\mathrm{SiB}_{3}$ equilibrium phase compared to the diamond structure of the Si parent phase, the direct nucleation of the equilibrium phase appears difficult if not impossible. Instead, precipitation of this phase should proceeds through the formation of coherent isostructural boron-enriched clusters having the structure of the parent silicon phase. Experiments indeed showed the formation of dilute boron- 


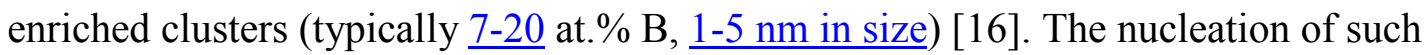
boron-enriched clusters at supersaturations and temperatures corresponding to the experimental conditions was modelled. The solubility limit of boron in silicon $\underline{\mathrm{c}} \underline{\alpha} \underline{\text { is }}$ close to $10^{-4}$ at $873 \mathrm{~K}$. In a regular solution model, the critical temperature is given by equation (12), $\mathrm{T}_{\underline{c}} \sim 4000 \mathrm{~K}$. This corresponds to $\mathrm{T} / \mathrm{T}_{\underline{c}} \sim 0.22(\mathrm{~T}=873 \mathrm{~K})$. For a nominal composition $\mathrm{c}_{0}$ of $1 \%$, the minimum value $\mathrm{c}_{\mathrm{m}}$ of the critical composition is $\sim 0.3$. The computed value of the composition $\mathrm{c}^{*}$ of the critical cluster is $\sim 0.63$ (driving force for nucleation $(\Delta \mathrm{f})$ close to $\left.7.5^{*} 10^{8} \mathrm{~J} / \mathrm{m}^{3}\right)$. The critical radius $\mathrm{R}^{*}$ was found close to $0.5 \mathrm{~nm}$

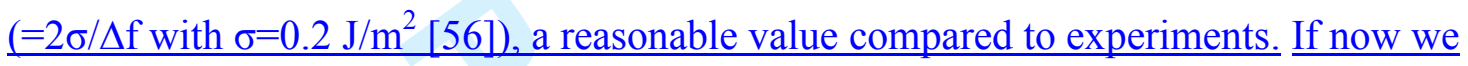
take a rather high elastic energy (misfit of 10\% [16]), critical clusters become almost pure in boron $\left(\mathrm{c}^{*} \sim 0.96\right)$. The effective driving force (i.e. taking into account elastic energy) was found lower $\left(\sim 6.3 * 10^{8} \mathrm{~J} / \mathrm{m}^{3}\right)$. The nucleation barrier therefore increases in comparison to the zero misfit case (as in Fig.2) but it remains lower than that computed for lower solute content with a misfit of $10 \%$. Thermodynamics therefore cannot thoroughly explain the formation of such dilute nuclei (less than $10 \%$ for R 1-2 nm) observed during the early stages of precipitation in boron implanted silicon. Kinetics arguments should also be taken into account to explain the formation of dilute nuclei. Diffusion mechanisms, particularly boron interactions with self-interstitials should be studied in more details in order to clarify the role of kinetics in the nucleation process in this system. Note that artefacts may also affect composition measured by atom probe tomography (APT). Local magnification effects may lead to underestimate the solute content in very small nuclei [57]. This can be particularly pronounced for SiB as boron is known to have a high evaporation field (defocusing effects in APT images [57]). 
The early stages of precipitation in binary $\mathrm{Fe}-\mathrm{Cr}$ alloy for a nominal composition $\left(\mathrm{c}_{0}\right)$ below the spinodal line were recently investigated [52]. Using atom probe tomography, Novy et al. showed that the composition of the Cr-enriched precipitates

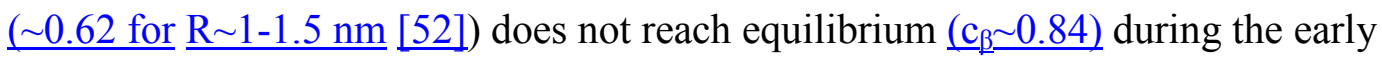
stages of precipitation. This was modelled in the same conditions as this experimental work (i.e. $\mathrm{T}=773 \mathrm{~K}, \mathrm{c}_{0}=0.2$ ). Instead of a regular solution model, we have used the rather complex free energy function given by Bonny et al. [see appendix of ref 58] so that to compute the driving force for nucleation as a function of the nucleus composition. This gives a solubility limit $\mathrm{c}_{\alpha}$ close to $15 \%$ at $773 \mathrm{~K}$ (in good agreement with experimental phase diagram) and the spinodal is $\mathrm{c}_{\mathrm{s}} \sim 34 \%$. The critical nucleus composition $\mathrm{c}^{*}$ was found equal to 0.78 (eq.8 neglecting the elastic energy). The addition of a low elastic energy (typically a misfit of $1 \%$ ) does not change $\mathrm{c}^{*}$ because it is negligible in comparison to the driving force. The critical nucleus composition $\mathrm{c}^{*}$ get closer to the equilibrium state ( $\left.\underline{\mathrm{c}}_{\beta} \sim 0.84\right)$ than that observed by Novy et al. (c $\sim 0.62$ for $\underline{\mathrm{R} \sim 1-1.5 \mathrm{~nm}}$ [52]). The corresponding driving force for nucleation $(\Delta \mathrm{f})$ is close to $3 * 10^{8} \mathrm{~J} / \mathrm{m}^{3}$. The critical radius $\mathrm{R}^{*}$ is thus close to $1 \mathrm{~nm}\left(=2 \sigma / \Delta \mathrm{f}\right.$ with $\left.\sigma=0.15 \mathrm{~J} / \mathrm{m}^{2}[59]\right)$. Our model based only on thermodynamics considerations shows the good trend but again it does

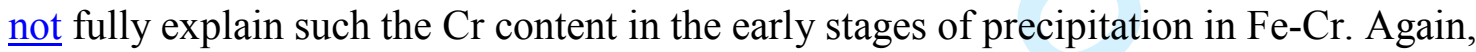
kinetics effects related to diffusion should play a role.

Precipitation of $\mathrm{Cu}$ (FCC) in the low temperature $\mathrm{BCC}$ phase of $\mathrm{Fe}$ is known to proceed via the nucleation of transient coherent embryo that have the same BCC structure as the parent $\mathrm{Fe}$ phase. The composition of such bcc $\mathrm{Cu}-\mathrm{Fe}$ precipitates is still a controversial issue [47]. One source of this is that composition data estimated using APT may be biased because of the occurrence of already mentioned local magnification 
effects. Copper is indeed known to have a lower evaporation field compared to iron (focusing effects). Consequently, iron coming from the matrix may fall in the precipitate $\underline{\text { region in APT images. This may artificially increase its iron content (i.e. lower } \mathrm{Cu}-}$ content). The influence of these effects has been studied theoretically and simulations predict them to be fairly small [57]. After thermal annealing or neutron or electronirradiation, a substantial fraction of Fe was detected in precipitates in the early stages, typically form 50 to 20 at.\% (i.e. $50-80$ at. $\% \mathrm{Cu}$ for $\mathrm{R}=1-2 \mathrm{~nm}$ ) [47-51]. Various interpretations accounting for the substantial amounts of $\mathrm{Fe}$ observed in $\mathrm{Cu}$ precipitates were proposed such as coherency stresses that might stabilize the precipitates or kinetic considerations [60]. We have applied the present model to the $\mathrm{Fe}-\mathrm{Cu}$ system in order to establish whether thermodynamics considerations can account at least partly for the nucleation of clusters containing less than 100 at. $\%$ of $\mathrm{Cu}$, the expected equilibrium value. We have considered the $\mathrm{Fe}-\mathrm{Cu}$ system at $773 \mathrm{~K}$ and for a nominal composition $c_{0}=0.015$. The free energy curve, $f(c)$, was obtained from Thermo-Calc $\mathbb{R}[61]$. The corresponding solubility limit is close to $10^{-4}$. The minimum value $\mathrm{c}_{\mathrm{m}}$ of the critical composition is $\sim 0.4$. Equation (8) (no misfit) gives a critical composition in copper, $\mathrm{c}^{*}$, equal to 0.75 . As in Fe-Cr, the elastic energy addition (misfit of 1\%) does not change drastically $\mathrm{c}^{*}$. The driving force for nucleation $(\Delta \mathrm{f})$ is close to $2.2 * 10^{9} \mathrm{~J} / \mathrm{m}^{3}$ (no misfit). The critical radius $\mathrm{R}^{*}$ is thus close to $0.25 \mathrm{~nm}\left(=2 \sigma / \Delta \mathrm{f}\right.$ with $\sigma=0.28 \mathrm{~J} / \mathrm{m}^{2}$ derived from the nearest neighbour broken bond model [62]). This time, this suggests that thermodynamically it is easier to form a critical nucleus with a much lower $\mathrm{Cu}$-content than that of the equilibrium phase [47-51]. Nagano et al. have simulated the composition of $\mathrm{Cu}$ particles formed during isothermal aging in bcc $\mathrm{Fe}-\mathrm{Cu}$ alloys using the Cahn-Hilliard nucleation theory and non-linear diffusion equation [63]. They found that whereas $\mathrm{Cu}$ nuclei are almost pure $\mathrm{Cu}$ in low $\mathrm{Cu}$ content alloys $(<1 \mathrm{at} . \% \mathrm{Cu})$, the 
$\mathrm{Cu}$ concentration in the nucleus is significantly less than unity in higher $\mathrm{Cu}$ content alloys ( $>1$ at. $\% \mathrm{Cu})$. Results derived from the present theory are in good agreement with the work of Nagano et al. as we found $\mathrm{c}^{*}=0.75$ for a high $\mathrm{Cu}$ content alloys $(1.5$ at.\%). The minimum energy pathway during nucleation was computed using a steepest descent method along the nucleation barrier and is shown in Fig.7. The black dashed line indicates the nucleation pathway in the present theory. This pathway passes through the saddle point $(\mathrm{c}=0.75, \mathrm{r}=1)$. The nucleation barrier is overestimated in the classical nucleation theory (grey trajectory in Fig. 7 is a vertical line located at $\mathrm{c}=1$ ). It was found that the equilibrium composition $(\mathrm{c} \sim 1)$ is only reached when the radius approaches $1 \mathrm{~nm}\left(\sim 1.5 \mathrm{R}^{*}\right)$. This indicates that even if nuclei are fairly dilute, they rapidly enrich up to the equilibrium. This behaviour is similar to the evolution of the nucleus composition simulated by Nagano et al. [63]. They found that during aging the nucleus composition sharply increases up to unity without any significant change in size. The particle growth then proceeds. This model predicts (for $\mathrm{c}_{0}=0.015$ and $\mathrm{T}=773$

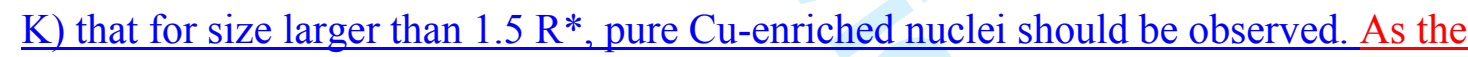
$\underline{\text { critical radius is small }(<0.5 \mathrm{~nm}) \text {, the present approach does not explain the large iron }}$ content observed in larger precipitates (typically $\mathrm{R}>2 \mathrm{~nm}$ ) observed by atom probe $\underline{\text { methods in similar conditions [47-51]. }}$

\section{Conclusion}

This paper has dealt with the non-classical nucleation of coherent precipitates that have a solute content lower than the "equilibrium" phase. This issue not only concerns the early stages of coherent precipitation (e.g. FeCr) but also the nucleation of transient coherent phases preceding the precipitation of incoherent phases (e.g. $\mathrm{FeCu}$, $\mathrm{SiB})$. The generalized Gibbs model developed by Schmelzer et al. [35] has been 
extended so that to include the influence of the elastic energy originating from the lattice misfit between nuclei and the parent phase. Isotropic elastic energy related to misfit has been considered. The composition of critical nuclei was determined through the minimization of the nucleation barrier in the radius (R) and composition (c) space.

Properties of critical nucleus $\left(\mathrm{R}^{*}, \mathrm{c}^{*}\right)$ were derived from the saddle point in the $R, c$ space for a regular solid solution $\left(T / T_{c}=0.3\right)$ and investigated as a function of the supersaturation. The model confirms that the classical nucleation theory is only valid for small supersaturation. Surprisingly, it was demonstrated that in the present theory the critical composition is independent on the interfacial coefficient that controls the dependence on the interfacial energy with the concentration gradient. The composition of critical nucleus was found to approach the matrix composition near the spinodal line. The critical radius of nucleation was found to diverge to infinity not only near the solubility limit $\mathrm{c}_{\alpha}$ but also close to the spinodal line $\mathrm{c}_{\mathrm{s}}$. The driving force and the nucleation barrier were both found to vanish near the spinodal line. The transition between the nucleation-growth and the spinodal regimes is found to be smooth in contrast to the classical nucleation theory.

The study of the influence of the elastic energy has shown non-anticipated effects. Paradoxically, increasing the elastic energy was found to increase the solute content in the critical nucleus. In spite of this non-classical behaviour, as expected, increasing the elastic energy increases both the nucleation barrier height and the critical radius. The minimum energy pathway was computed so that to predict the kinetic pathway of nucleation. We found that nucleation often proceeds with three step process. Nuclei grow without change in composition and then they rapidly enrich until the 
expected composition $\left(\mathrm{c}_{\beta}\right)$. Finally, they grow with the composition $\mathrm{c}_{\beta}$. However, in contrast to Schmelzer et al. [36], we found that this behaviour is not general. For small supersaturation, the change in cluster composition occurs sharply for very small radius and then the composition slowly increases with a significant change in size. The elastic energy was found to slow down the evolution of the nuclei composition with R.

Predictions were confronted to experiments. In good agreement with observations in dilute $\mathrm{Fe}-\mathrm{Cu}$ alloys, this model shows that nuclei have a $\mathrm{Cu}$ content lower than the expected BCC phase (pure $\mathrm{Cu}$ ). In agreement with Nagano et al. [63] we found that even if nuclei are fairly dilute, they rapidly enrich up to the equilibrium composition without a significant change in size. This thermodynamics approach however fails to fully account observations in Si-B and Fe-Cr. Kinetics effects related to diffusion should play a role. Monte carlo simulations are here of great interest to elucidate the role of diffusion in kinetics of precipitation [64-69]. It should be kept in mind that the results derived from the present theory are strongly dependent on the shape of the free energy curve $f(c)$. More realistic expressions of $f(c)$ based on first principles computations should lead to better agreement with experiments.

Acknowledgements The authors acknowledge C. Pareige, B. Radiguet (GPM, Rouen) and G. Martin (CEA, Saclay) for fruitful discussion. The first author also acknowledges N. Masquelier for his help in the use of the Thermo-Calc ${ }^{\circledR}$ software.

\section{References}


[1] J.W. Gibbs, Trans. Connecticut Acad. Sci. 3 (108) (1875-1878) 343, see also: J.W. Gibbs, The Collected Works, vol. 1, Longmans Green, New York/London/Toronto, 1928.

[2] J.D. van der Waals, Ph. Kohnstamm, Lehrbuch der Thermodynamik, JohannAmbrosius-Barth Verlag, Leipzig/Amsterdam, 1908.

[3] J.S. Rowlinson, Translation of J.D. van der Waals, J. Stat. Phys. 20 (1979) 197, German version: J.D. Van der Waals, Z. Phys. Chem. 13 (1893) 657.

[4] M. Volmer, Kinetik der Phasenbildung, Steinkopff, Dresden/Leipzig, 1939.

[5] Ya.I. Frenkel, Kinetic Theory of Liquids, Oxford Univ. Press, Oxford, 1946.

[6] A.C. Zettlemoyer (Ed.), Nucleation, Decker, New York, 1969.

[7] A.C. Zettlemoyer, Adv. Colloid Interface Science 7 (1977).

[8] J.D. Gunton, M. San Miguel, P.S. Sahni, in: C. Domb, J.L. Lebowitz (Eds.), Phase Transitions and Critical Phenomena, Vol. 8, Academic Press, London/New York, 1983.

[9] V.P. Skripov, Metastable Liquids, Nauka, Moscow, 1972, Wiley, New York, 1974 [in Russian].

[10] J.W. Cahn, J.E. Hilliard, J. Chem. Phys. 28 (1959) 258.

[11] J.W. Cahn, J.E. Hilliard, J. Chem. Phys. 31 (1959) 688.

[12] R. Becker and W. Döring. Ann. Phys. (Leipzig) 24, 719-752 (1935)

[13] Zeldovich, J. Exper. Theor. Phys. U.S.S.R. 12, 525 (1942)

[14] D. Turnbull, J. C. Fisher, J. Chem. Phys. 17 (1949) 71.

[15] T. Hashizume, K. Hono, Y. Hasegawa, K. Hirano et T. Sakurai, J. Phys. Colloques 47 (1986) C2-171-C2-177.

[16] O. Cojocaru-Mirédin, D. Mangelinck and D. Blavette, J. of Appl. Phys. 106 (2009) 113525.

[17] S. Duguay, F. Vurpillot, T. Philippe, E. Cadel, R. Lardé, B. Deconihout, G. Servanton, and R. Pantel, J. of Appl. Phys. 106 (2009) 106102. 
[18] D. Blavette, A. Bostel, J.M. Sarrau, B. Deconihout et A. Menand, Nature 363 (1993) 432.

[19] C. Pareige, F.Soisson, G. Martin and D. Blavette, Acta Mater. 47 (1999) 1889.

[20] E. Marquis, Microscopy and Microanalysis. 13 (2007) 484

[21] T. Al Kassab, P. Haasen, Z. fur Metallkunde 84 (1993) 248.

[22] B. Radiguet, D. Blavette, N. Wanderka, J. Banhart, K. L. Sahoo, Appl. Phys. Lett. $92(2008) 103126$.

[23] G. A. Edwards, K. Stiller, GL Dunlop, M. J. Couper, Acta mat. 46 (1998) 3893.

[24] M. Murayama and K. Hono, Acta mat. 47 (1999) 1537.

[25] S.P. Ringer, K Hono, I.J. Polmear, T. Sakurai, Acta mat. 44 (1996) 1883.

[26] A Heinrich, T Al-Kassab, R. Kircheim - Materials Science and engeneering A 353 (2003) 168.

[27] M.K. Miller, J.M. Hyde, M.G. Hetherington, A. Cerezo, G.D.W. Smith and C.M. Elliott, Acta Metall. Mater. 43 (1995) 3385.

[28] C.S.T.Chang,I.Wieler, N.Wanderka, J.Banhart, Ultramicroscopy 109 (2009) 585.

[29] M.E. Krug, D.C. Dunand and D.N. Seidman, Appl Phys Lett 92 (2008) 124107.

[30] C.K. Sudbrack, R.D. Noebe and D.N. Seidman, Phys Rev B 73 (2006) 212101.

[31] Z. Mao, C.K. Sudbrack, K.E. Yoon, G. Martin and D.N. Seidman, Nature Materials $6(2007) 210$.

[32] J. M. Roussel, P. bellon, Phys. Rev. B 63 (2001) 184114.

[33] A. Perini, G. Jacucci, G. Martin, Phys. Rev. B 29 (1984) 2689.

[34] T. T. Rautiainen, A. P. Sutton, Phys. Rev. B 59 (1999) 13681.

[35] J. W. P. Schmelzer, J. Schmelzer, I. Gutzow, J. Chem. Phys. 112 (2000) 3820.

[36] J. W. P. Schmelzer, A. R. Gokhman, V. M. Fokin, On the Dynamics of First-Order Phase Transitions in Multi-Component Systems, J. Colloid Interface Science, 272, 109 (2004).

[37] J. D. Eshelby, Solid State Physics, 3 (1956) 79. 
[38] J.W.P. Schmelzer, R. Müller, J. Möller, I. S. Gutzow, Journal of Non-Crystalline Solids 315 (2003) 144-160

[39] G. Henkelman and H. Jonsson, Improved tangent estimate in the nudged elastic band method for finding minimum energy paths and saddle points, J. Chem. Phys. 113 (2000), p. 9978.

[40] G. Henkelman, B. Uberuaga and H. Jónsson, A climbing image nudged elastic band method for finding saddle points and minimum energy paths, J. Chem. Phys. 113 (2000), pp. 9901-9904.

[41] W. E,W. Ren and E Vanden-Eijnden, Simplified and improved string method for computing the minimum energy paths in barrier-crossing events, J. Chem. Phys., 126, 164103, 2007.

[42] W. E, W. Ren and E Vanden-Eijnden, String method for the study of rare events, Phys. Rev. B, 66, 052301, (2002).

[43] L. Zhang, L.Q. Chen and Q. Du, J. of Comput. Phys. 229 (2010) 6574-6584.

[44] L. Zhang, L.Q. Chen and Q. Du, Morphology of critical nuclei in solid state phase transformations, Phys. Rev. Lett., 98, 265703 (2007).

[45] L. Zhang, L.Q. Chen and Q. Du, Diffuse-interface description of strain-dominated morphology of critical nuclei in phase transformations, Acta Materialia, 56 (2008), pp.3568-3576.

[46] L. Zhang, L.Q. Chen and Q. Du, Mathematical and Numerical Aspects of Phasefield Approach to Critical Morphology in Solids, J. Sci. Comput., 37 (2008), pp.89-102.

[47] M. E. Fine, J. Z. Liu, M. D. Asta, Material Science and Engineering A 463 (2007) 271-274.

[48] S. R. Goodman, S. S. Brenner, and J. R. Low, Metall. Trans. 4 (1973) 2371.

[49] P. Pareige, doctoral thesis, Université de Rouen, Rouen (1994).

[50] P. Auger, P. Pareige, M. Akamatesu and J.-C. Van Duysen, J. Nucl. Mater. 211 (1994) 194.

[51] M.K. Miller, B.D. Wirth, G.R. Odette, Materials Science and Engineering A353 (2003) 133-139. 
[52] S. Novy, P. Pareige and C. Pareige, J. Nucl. Mater. 384 (2009) 96.

[53] M. Ngamo, S. Duguay, F. Cristiano, Daoud-Ketata K, P. Pareige, J. of Appl. Phys. 105 (2009) 104904.

[54] T. Philippe, S. Duguay, J.J. Grob, D. Mathiot, and D. Blavette, Thin solid films 518 (2010) 2406-2408.

[55] T. Philippe, S. Duguay, D. Mathiot, and D. Blavette, J. of Appl. Phys. 109 (2011) 023501.

[56] S. Solmi, E. Landi, and F. Baruffaldi, J. Appl. Phys. 68 (1990) 3250.

[57] D. Blavette, F. Vurpillot, P. Pareige, A. Menand, Ultramicroscopy 89 (2001) 145153.

[58] G. Bonny, D. Terentyev, L. Malerba, Journal of Phase Equilibria and Diffusion 31 (2010) 439.

[59] S. Lu, Q-M Hu, B. Johansson, L. Vitos, Phys. Status Solidi B 1-4 (2011) / DOI $10.1002 /$ pssb.201147123.

[60] F. Soisson, A. Barbu, and G. Martin, Acta Mater. 44, (1996) 3789.

[61] Thermo-Calc® . http://www.thermocalc.com/software.htm. TCBIN database.

[62] Z.G. Yang, M. Enomoto, Mater. Sci. Eng. A 332 (2002) 184.

[63] T. Nagano and M. Enomoto, Scripta Materialia 55 (2006) 223-226.

[64] F. Soisson, G. Martin, Phys. Rev. B 62 (2000) 203.

[65] F. Soisson, Philos. Mag. 85 (2005) 489.

[66] C. Schmuck, P. Caron, A. Hauet, D. Blavette, Philos. Mag. A 76 (1997) 527-542.

[67] M. Athenes, P. Bellon, G. Martin, F. Haider, Acta Materialia, 44 (1996) 4739.

[68] M. Athenes, P. Bellon, G. Martin, Philos. Mag. A, 76 (1997) 565-585.

[69] M. Athenes, P. Bellon and G. Martin, Acta Materialia 48 (2000) 2675.

\section{Figure captions}


Fig.1 Schematic representation of the free energy curve for a system exhibiting isostructural decomposition.

Fig.2 Nuclei properties as a function of the nominal composition $\mathrm{c}_{0}$ for a reduced temperature equal to 0.3. Composition of the critical cluster $\mathrm{c}^{*}$ (a), driving force $\Gamma(\mathrm{b})$, nucleation barrier $\mathrm{W}(\mathrm{c})$, critical radius $\mathrm{R}^{*}(\mathrm{~d})$. The influence of the elastic energy due to lattice misfit between phases is also shown for various elastic constants $(\mathrm{k}=0,1,2)$.

Fig.3 The reduced nucleation barrier $\Phi$ as a function of the nucleus size (reduced radius $\left.\mathrm{r}=\mathrm{R} / \mathrm{R}^{*}\right)$ and composition (c) for $\eta=0.3$, and a nominal composition $\mathrm{c}_{0}=0.04(\mathrm{k}=0)$. The saddle point is localized at $\mathrm{r}=1$ and $\mathrm{c}=\mathrm{c}^{*}=0.456$.

Fig.4 Evolution of the solute content in nuclei (c) as a function of their size (R) for various solute concentration $\left(c_{0}=0.01-0.07, \eta=0.3\right)$ and for no lattice misfit $(k=0)$.

Fig.5 Evolution of the solute content in nuclei (c) as a function of their size (R) for various elastic constant $(\mathrm{k}=0,1,2$ and $\eta=0.3)$. The nominal composition was $\mathrm{c}_{0}=0.04$.

Fig.6 Evolution of the solute content in nuclei (c) as a function of their size (R) for various elastic constant $(\mathrm{k}=0,1,2$ and $\eta=0.3)$. The nominal composition was $\mathrm{c}_{0}=0.02$.

Fig.7 Nucleation pathway in the $(\mathrm{r}, \mathrm{c})$ space for the precipitation of $\mathrm{Cu}$ in $\mathrm{FeCu}(0.015$ $\mathrm{Cu}, \mathrm{T}=773 \mathrm{~K}$ ). In the classical nucleation theory (grey trajectory) the nucleus composition $\mathrm{c}$ is constant and equal to $\mathrm{c}=1$, the equilibrium concentration as given by the phase diagram. 


1
2
3
4
5
6
7
8
9
10
11
12
13
14
15
16
17
18
19
20
21
22
23
24
25
26
27
28
29
30
31
32
33
34
35
36
37
38
39
40
41
42
43
40
45
49
50
51
52
53
55
50

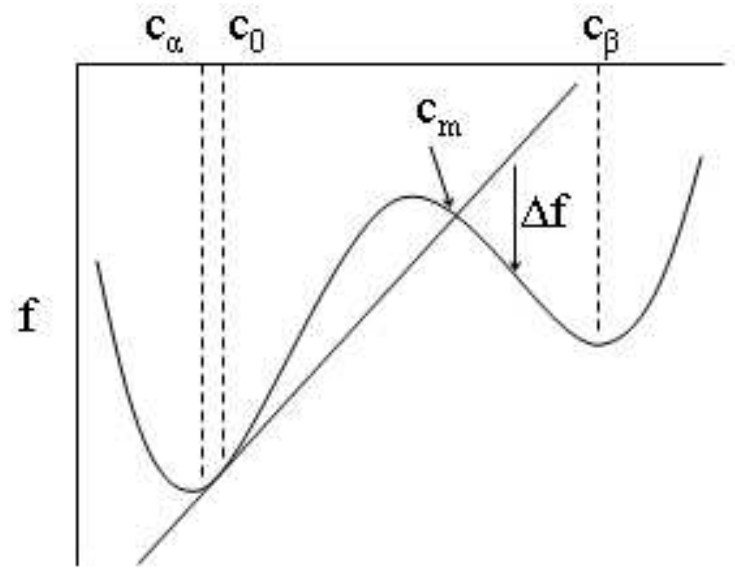

$76 \times 60 \mathrm{~mm}(96 \times 96 \mathrm{DPI})$

http://mc.manuscriptcentral.com/pm-pml 
a)

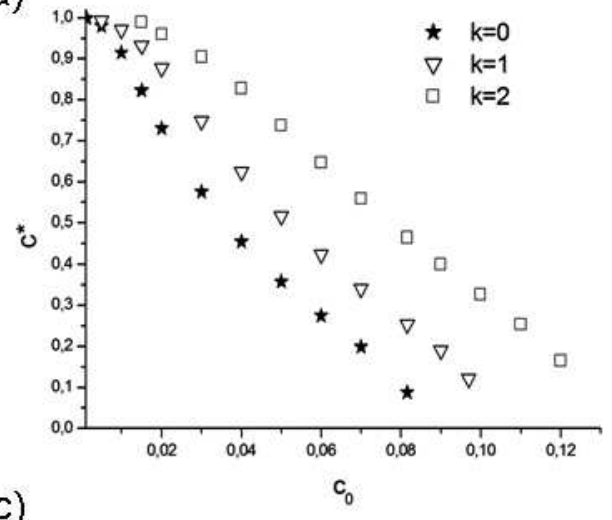

c)

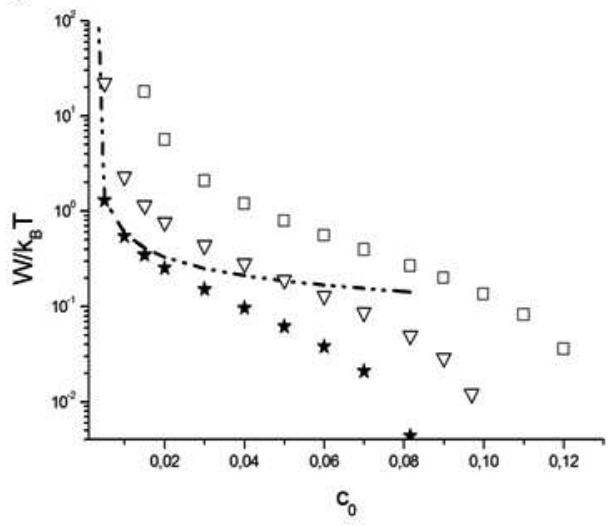

b)

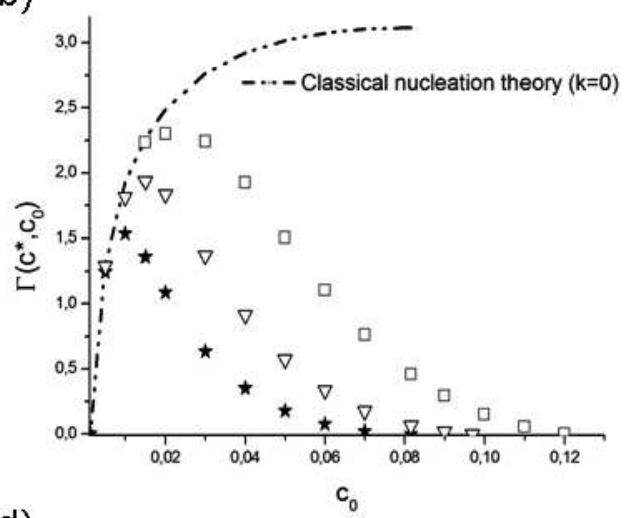

d)

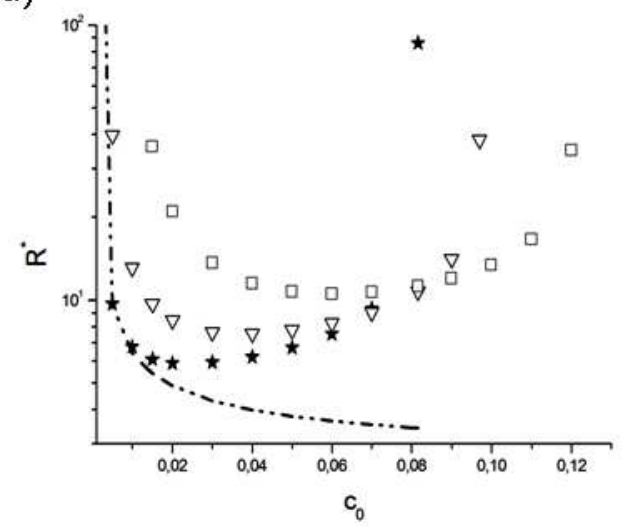

$207 \times 176 \mathrm{~mm}(96 \times 96 \mathrm{DPI})$

http://mc.manuscriptcentral.com/pm-pml 


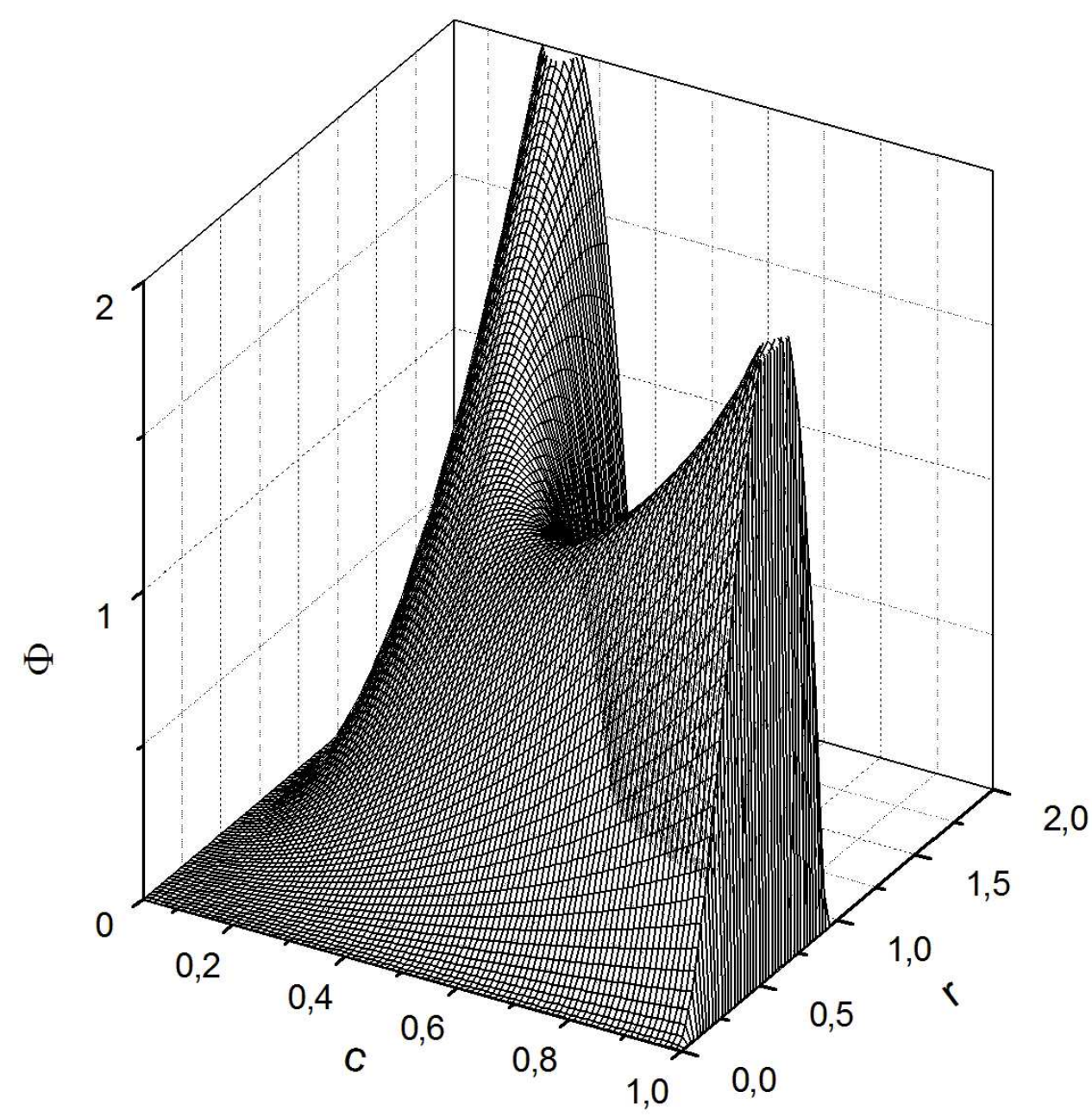

$197 \times 198 m m(150 \times 150$ DPI $)$ 


1
2
3
4
5
6
7
8
9
10
11
12
13
14
15
16
17
18
19
20
21
22
23
24
25
26
27
28
29
30
31
32
33
34
35
36
37
38
39
40
41
42
43
44
45
46
47
48
49
50
51
52
53
54
55
56
57
59
60

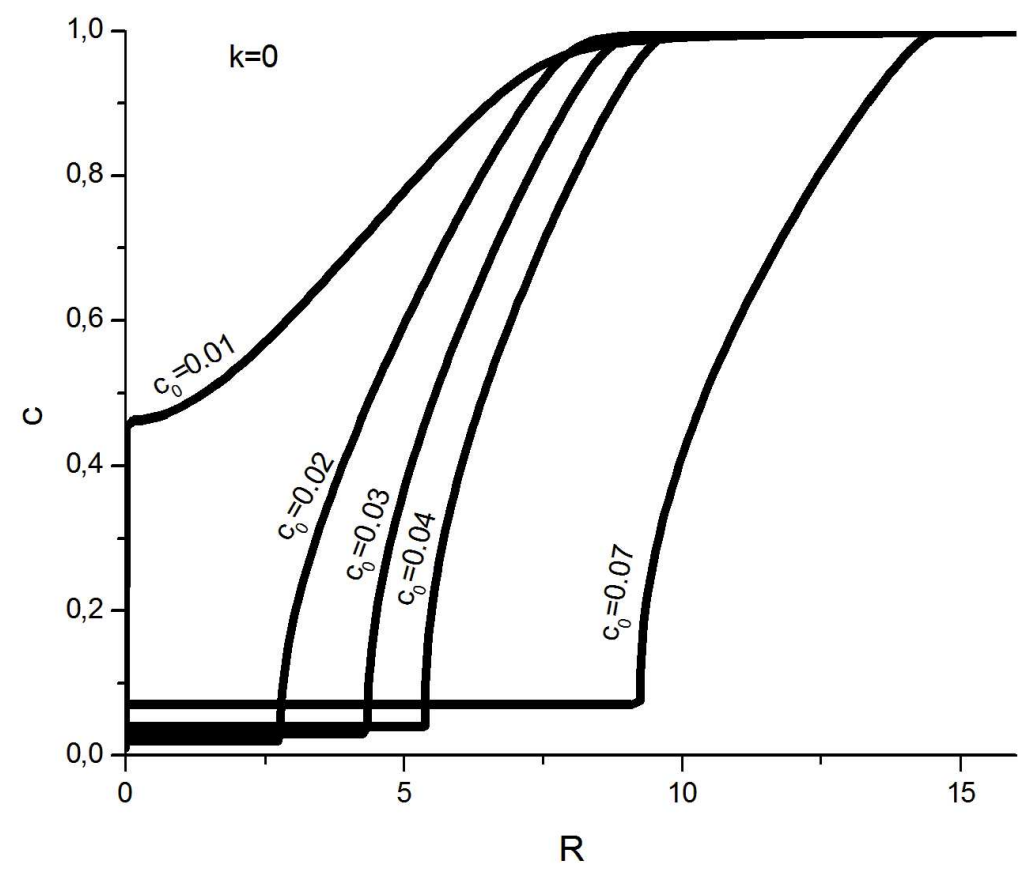

$279 \times 215 \mathrm{~mm}(150 \times 150 \mathrm{DPI})$ 


1
2
3
4
5
6
7
8
9
10
11
12
13
14
15
16
17
18
19
20
21
22
23
24
25
26
27
28
29
30
31
32
33
34
35
36
37
38
39
40
41
42
43
44
45
46
47
48
49
50
51
52
53
54
55
56
57
58
60

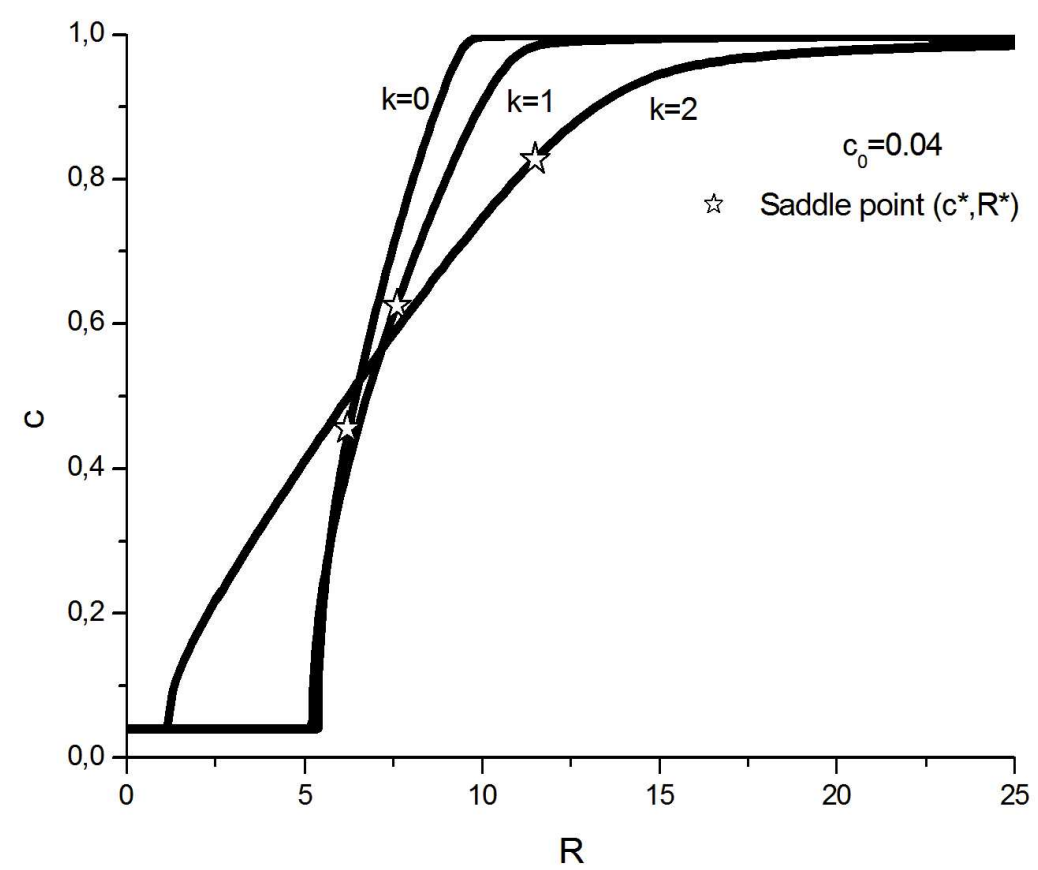

$279 \times 215 \mathrm{~mm}(150 \times 150 \mathrm{DPI})$ 


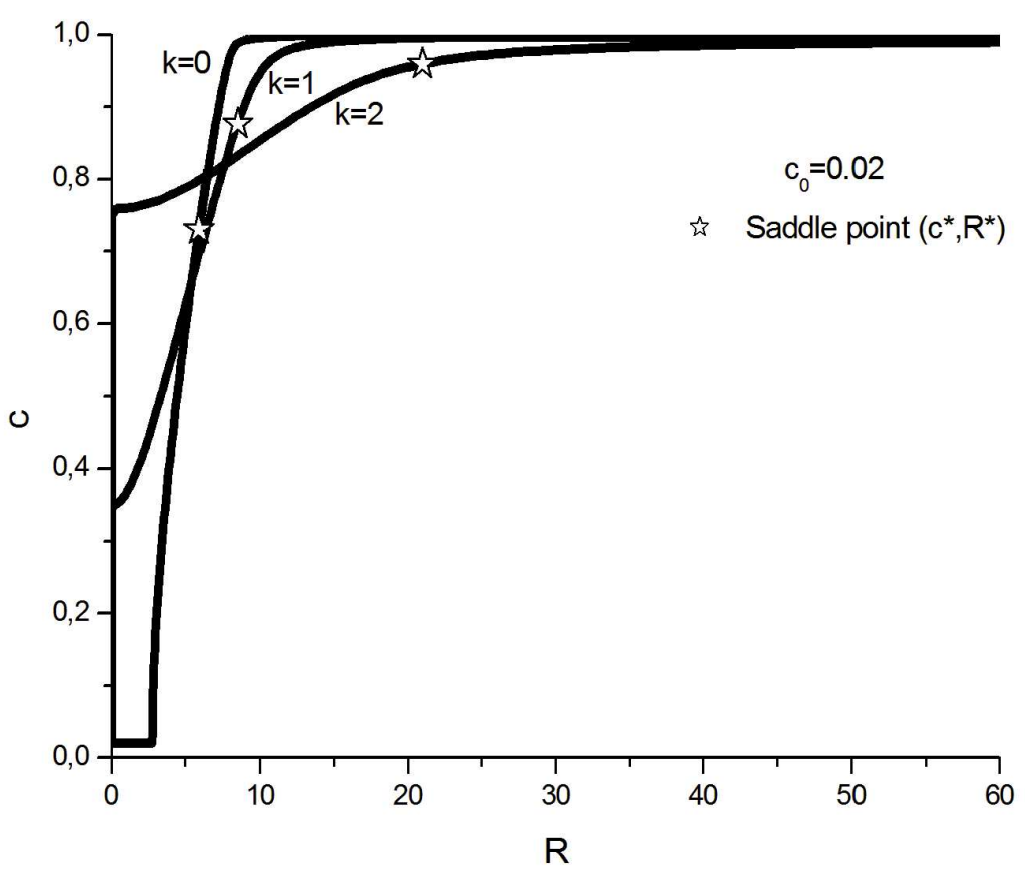

$279 \times 215 \mathrm{~mm}(150 \times 150 \mathrm{DPI})$ 


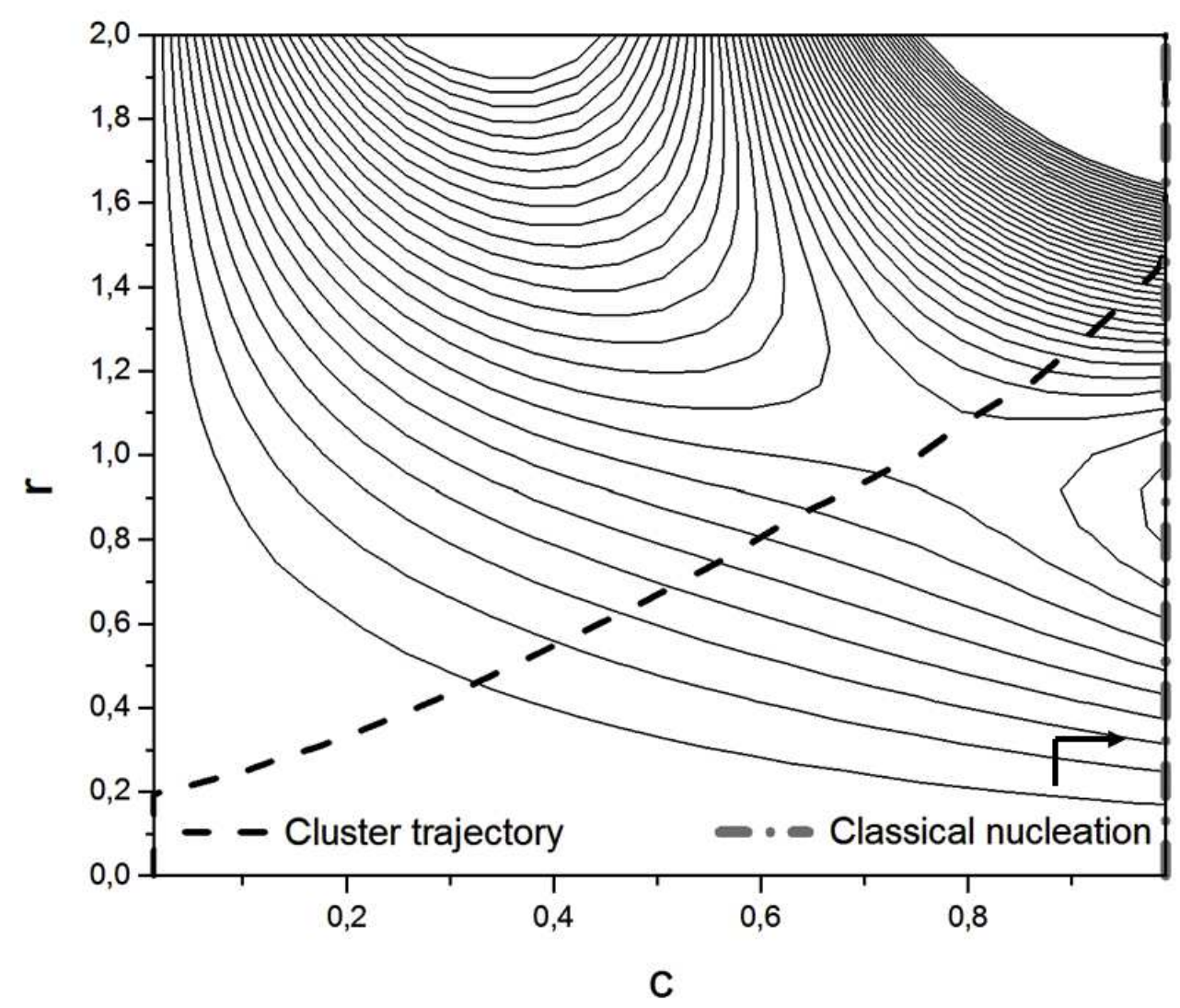

$215 \times 183 \mathrm{~mm}(96 \times 96 \mathrm{DPI})$ 